\title{
$v$-Process in Population III Core-Collapse Supernovae
}

\section{Takashi Yoshida*}

Division of Theoretical Astronomy, National Astronomical Observatory of Japan

E-mail: takashi.yoshidaenao.ac.jp

\section{Hideyuki Umeda}

Department of Astronomy, School of Science, University of Tokyo

E-mail: umeda@astron.s.u-tokyo.ac.jp

\section{Ken'ichi Nomoto}

Department of Astronomy, School of Science, University of Tokyo

Institute for the Physics and Mathematics of the Universe, University of Tokyo

E-mail: nomoto@astron.s.u-tokyo.ac.jp

We investigate the effects of the $v$-process on the production of odd- $Z$ iron-peak elements in the supernovae of 15 and $25 M_{\odot}$ Population III stars and the hypernova of a $25 M_{\odot}$ Population III star. The abundances of $\mathrm{Sc}, \mathrm{Mn}$, and Co produced in complete and incomplete Si burning regions are enhanced through the $v$-process and subsequent neutron- and proton-capture reactions. The enhanced yields depend on the total energy released by neutrinos. The $\mathrm{Mn} / \mathrm{Fe}$ ratios of the 15 and $25 M_{\odot}$ supernovae with the $v$-process and an adequate total neutrino energy are in the range observed in low mass extremely metal-poor halo stars. On the other hand, the yields of Sc and Co are still small to explain the observed ratios to Fe.

10th Symposium on Nuclei in the Cosmos

July 27 - August 12008

Mackinac Island, Michigan, USA

\footnotetext{
* Speaker.
} 


\section{Introduction}

Recent observations with high resolution spectroscopy have revealed the abundance distributions of low-mass extremely metal-poor (EMP) stars and very metal-poor (VMP) stars. The observed abundance ratios to Fe averaged over these EMP and VMP stars have small scatters from the corresponding solar ratios; the scatters are within 0.5 dex for most elements [1]. The observed abundance ratios are considered to relate to the nucleosynthesis of Population III supernovae and hypernovae (e.g., [2]) and early stage of the Galactic chemical evolution [3]. Theoretical evaluation of the yields in Population III supernovae and hypernovae [丹] showed that the abundance ratios of $\mathrm{C}, \mathrm{Na}, \mathrm{Mg}, \mathrm{Al}, \mathrm{Si}, \mathrm{Ca}, \mathrm{Ni}$, and $\mathrm{Zn}$ to Fe observed in EMP stars agree well with those of Population III hypernovae. The observed abundance pattern for $\mathrm{C}, \mathrm{O}, \mathrm{Na}, \mathrm{Mg}, \mathrm{Al}, \mathrm{Si}, \mathrm{Ca}, \mathrm{Cr}, \mathrm{Ni}$, and $\mathrm{Zn}$ in VMP stars are explained by the yields from Population III supernovae and hypernovae integrated over the Salpeter initial mass function. However, the yields of N, K, Sc, Ti, Mn, and Co in the supernovae and hypernovae are still too small to explain the observed ratios.

The nucleosynthesis induced by neutrino-nucleus interactions, the $v$-process, is one of the synthesis processes in supernovae [5]. The yields of some rare elements such as $\mathrm{Li}, \mathrm{B}$, and $\mathrm{F}$ are enhanced by the $v$-process [6, ㄱ, 8, 勿]. Therefore, the $v$-process is expected to increase the yields of some odd- $Z$ elements. In this study, we investigate the effects of the $v$-process on the production of odd-Z iron-peak elements, Sc, Mn, and Co, in Population III supernovae and hypernova. We compare the evaluated abundance patterns of the supernovae and hypernova taking into account the $v$-process to those observed in EMP stars. Detailed results are explained in [10].

\section{Supernova models}

We adopt 15 and $25 M_{\odot}$ Population III star models in [11]. The supernova explosions of the 15 and $25 M_{\odot}$ stars are calculated with the explosion energy of $1 \times 10^{51}$ ergs. The hypernova of the $25 M_{\odot}$ is also calculated with the explosion energy of $2 \times 10^{52}$ ergs. We assume the spherical explosions. Detailed nucleosynthesis calculations of the supernovae and hypernova are carried out by postprocessing with a nuclear reaction network of 281 species of nuclei. The mixing-fallback effect (e.g. [12]) is taken into account for the hypernova model. The $v$-process reactions in the network are taken from [13].

In order to calculate the $v$-process, we assume the supernova neutrinos as follows. The neutrino luminosity decreases exponentially with a time scale of $\tau_{v}=3 \mathrm{~s} \mathrm{(after} \mathrm{[5]).} \mathrm{The} \mathrm{total} \mathrm{energy}$ released by neutrinos are parameterized to be $E_{v}=3 \times 10^{53} \mathrm{ergs}$ (the standard value), $9 \times 10^{53}$ ergs, and $3 \times 10^{54}$ ergs. The energy is equipartitioned in all flavors of neutrinos and antineutrinos. The neutrino energy spectra follow Fermi-Dirac distributions with zero chemical potentials. The neutrino temperatures are set to be $4 \mathrm{MeV}, 4 \mathrm{MeV}$, and $6 \mathrm{MeV}$ for $v_{e}, \bar{v}_{e}$, and $v_{\mu, \tau}$ and $\bar{v}_{\mu, \tau}$ [13].

\section{Results}

First, we explain the main production reactions of Sc, Mn, and Co in the supernovae and hypernova. When the $v$-process is not taken into account, most of Sc is produced through incomplete Si burning independent of the stellar mass. Sc is almost exhausted in complete Si burning region. 
Most of $\mathrm{Mn}$ is also produced through incomplete $\mathrm{Si}$ burning. It is mainly produced as ${ }^{55} \mathrm{Co}$. The main production processes are ${ }^{54} \mathrm{Fe}(p, \gamma){ }^{55} \mathrm{Co}$ and ${ }^{56} \mathrm{Ni}(\gamma, p){ }^{55} \mathrm{Co}$. A part of $\mathrm{Mn}$ is produced in complete Si burning region, too. However, the produced amount is much smaller in the case of supernovae. Most of Co is produced through complete Si burning.

When the $v$-process is taken into account, the mass fractions of Sc, Mn, and Co are enhanced. The mass fraction of $\mathrm{Sc}$ is enhanced in complete $\mathrm{Si}$ burning region and partly in incomplete $\mathrm{Si}$ burning region. Most of $\mathrm{Sc}$ is produced through ${ }^{44} \mathrm{Ti}(p, \gamma)^{45} \mathrm{~V}\left(\beta^{+}\right)^{45} \mathrm{Ti}\left(\beta^{+}\right)^{45} \mathrm{Sc}$. The protons are supplied through ${ }^{56} \mathrm{Ni}\left(v, v^{\prime} p\right){ }^{55} \mathrm{Co}$ after the cease of complete Si burning.

The mass fraction of $\mathrm{Mn}$ in complete Si burning region increases by the $v$-process by about three orders of magnitude. The $\mathrm{Mn}$ is originally produced as ${ }^{55} \mathrm{Co}$ through the $v$-process reaction ${ }^{56} \mathrm{Ni}\left(v, v^{\prime} p\right){ }^{55} \mathrm{Co}$. Some additional $\mathrm{Mn}$ is produced through the $v$-process in incomplete Si burning region.

The $v$-process enhances the Co production in complete Si burning region. Most of Co is originally produced as ${ }^{59} \mathrm{Cu}$ and a small amount is produced as ${ }^{59} \mathrm{Ni}$. The main production processes of ${ }^{59} \mathrm{Co}$ and ${ }^{59} \mathrm{Ni}$ are ${ }^{58} \mathrm{Ni}(p, \gamma){ }^{59} \mathrm{Cu}$ and ${ }^{58} \mathrm{Ni}(n, \gamma){ }^{59} \mathrm{Ni}$, respectively. Protons and neutrons are produced through the $v$-process. The contribution of the $v$-process in the Co production is very small in the hypernova.

Next, we discuss the effects of the $v$-process on the abundance patterns of the Population III supernovae and hypernova. Figure 11 shows the abundance ratios $[\mathrm{X} / \mathrm{Fe}]$ of the Population III supernovae and hypernova as well as the observed abundance ratios averaged over 22 EMP stars with $[\mathrm{Fe} / \mathrm{H}] \leq-3.0$ in [1] $\left([\mathrm{X} / \mathrm{Y}] \equiv \log \left(N_{\mathrm{X}} / N_{\mathrm{Y}}\right)-\log \left(N_{\mathrm{X}} / N_{\mathrm{Y}}\right) \odot\right.$ where $N_{\mathrm{X}}$ and $N_{\mathrm{Y}}$ are the abundances of elements $\mathrm{X}$ and $\mathrm{Y}$ ). The abundance ratios of Sc, Mn, and Co to Fe are enhanced by the $v$-process in the supernovae. When the total neutrino energy is $3 \times 10^{53} \mathrm{ergs}$ (the standard value), the ratios of $\mathrm{Sc}, \mathrm{Mn}$, and $\mathrm{Co}$ to $\mathrm{Fe}$ in the supernovae increase by $0.25-0.64 \mathrm{dex}, 0.24-0.38$ dex, and $0.37-0.53$ dex, respectively. Therefore, more than $40 \%$ of the yields are produced through the $v$-process. On the other hand, these ratios are enhanced by $0.77-1.44 \mathrm{dex}, 1.11-1.39$ dex, and $1.26-1.38 \mathrm{dex}$ in the case of the strongest neutrino irradiation. Most of the elements increase by more than one order of magnitude by the $v$-process.

The increase in the abundance ratios of $\mathrm{Sc}$ and $\mathrm{Co}$ to $\mathrm{Fe}$ is smaller in the hypernova. The $\mathrm{Sc} / \mathrm{Fe}$ ratio increases by 0.13 and $0.69 \mathrm{dex}$ with the $v$-process and $E_{v}=3 \times 10^{53}$ and $3 \times 10^{54}$ ergs, respectively. The $\mathrm{Co} / \mathrm{Fe}$ ratio does not increase by the $v$-process. When the $v$-process is not considered, the abundance ratios of $\mathrm{Sc}$ and $\mathrm{Co}$ to $\mathrm{Fe}$ in the hypernova are larger than those of the supernovae. The hypernova produces larger amounts of Sc and Co through complete and incomplete Si burnings. The $\mathrm{Mn} / \mathrm{Fe}$ ratio increases by the $v$-process in the hypernovae. However, the increase is smaller than that in the supernovae since larger amount of Fe is produced in complete Si burning region.

We compare the abundance ratios of $\mathrm{Sc}, \mathrm{Mn}$, and $\mathrm{Co}$ to $\mathrm{Fe}$ with the average ratios observed in the EMP stars [1]]. We see that the $\mathrm{Mn} / \mathrm{Fe}$ ratio in the supernovae is above or below the lower limit of the observed ratios, when the $v$-process is not considered. On the other hand, this ratio is within the range of the observed ratio, when the $v$-process is taken into account and the total neutrino energy is $\sim(3-9) \times 10^{53}$ ergs. Therefore, the $v$-process in Population III supernovae is one of the preferable processes of Mn observed in EMP stars. The hypernova also reproduces the observed ratio but the strongest neutrino irradiation is necessary. The $\mathrm{Sc} / \mathrm{Fe}$ ratio in the supernovae and hypernova is 

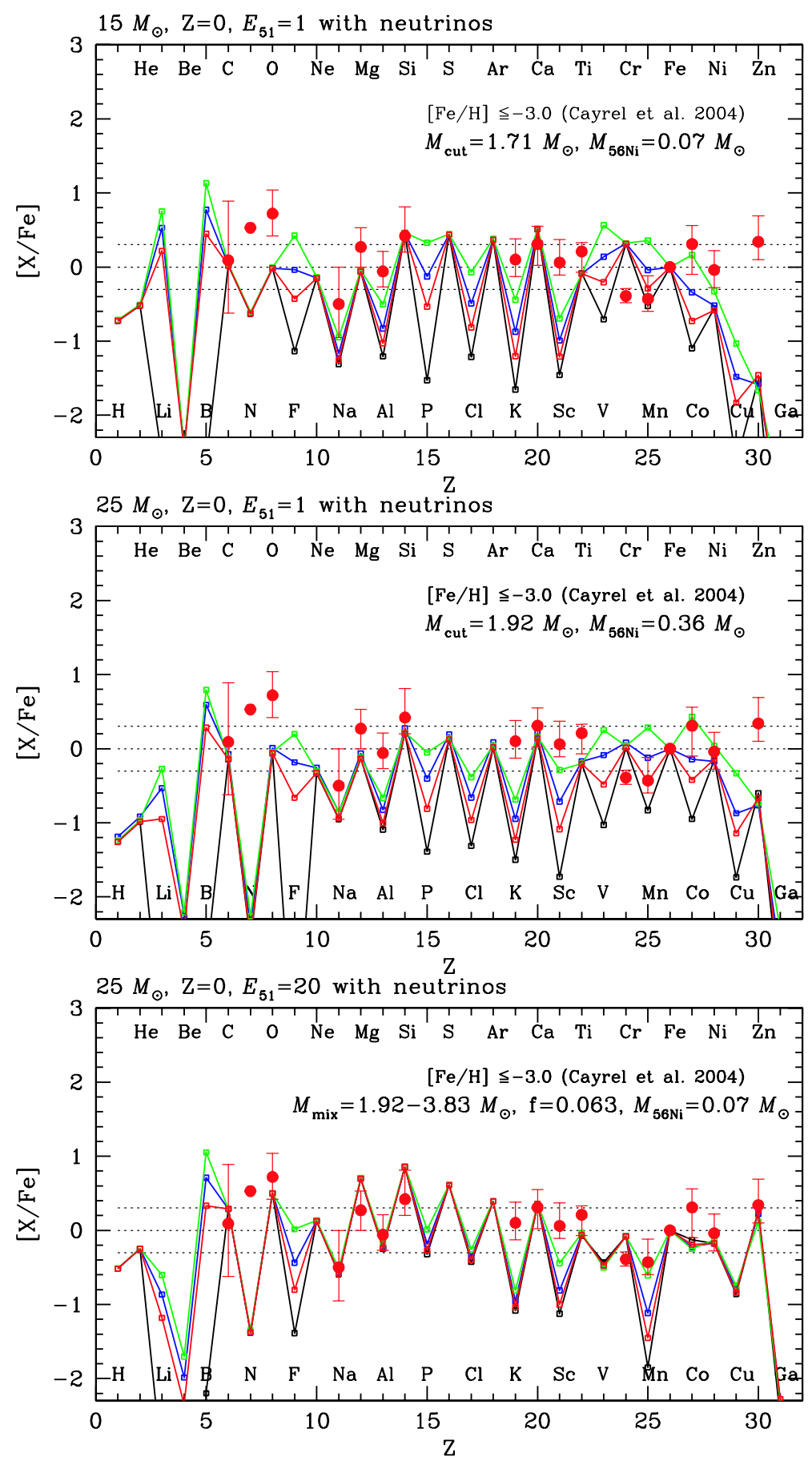

Figure 1: The abundance ratios to $\mathrm{Fe}[\mathrm{X} / \mathrm{Fe}]$ in the supernovae of $15 M_{\odot}$ Population III star (top panel) and $25 M_{\odot}$ star (middle panel) and the hypernova of $25 M_{\odot}$ Population III star (bottom panel). Black lines correspond to the ratios when the $v$-process is not taken into account. Red, blue, and green lines are the ratios with the $v$-process and $E_{v}=3 \times 10^{53}, 9 \times 10^{53}$, and $3 \times 10^{54} \mathrm{ergs}$, respectively. Circles with error bars indicate the abundance ratios averaged over $22 \mathrm{EMP}$ stars with $[\mathrm{Fe} / \mathrm{H}] \leq-3.0$ [1]. 
smaller than the observed ratio even with the $v$-process and the strongest neutrino irradiation. Thus, other production processes to produce $\mathrm{Sc}$ should be considered. Recently, preferable processes are considered to be the $v p$-process [14] and aspherical supernova explosions [15]. The $\mathrm{Co} / \mathrm{Fe}$ ratio in the supernovae reproduces the observed ratio. However, strong neutrino irradiation with $E_{v}>9 \times 10^{53}$ ergs is necessary. Hypernovae are considered to be one of the production sites of Co observed in EMP stars. When the electron fraction $Y_{e}$ in complete Si burning region is slightly larger than 0.5 , a suitable amount of Co is produced [12].

\section{Summary}

We investigated the effects of the $v$-process on the production of Sc, Mn, and Co in Population III supernovae and hypernovae. These elements are mainly produced in complete and incomplete $\mathrm{Si}$ burning regions. The main production process of the $v$-process is ${ }^{56} \mathrm{Ni}\left(v, v^{\prime} p\right)^{55} \mathrm{Co}$, through which $\mathrm{Mn}$ is produced. Capture reactions of protons and neutrons produced through the $v$-process are also important to enhance the yields of Sc and Co. The $\mathrm{Mn} / \mathrm{Fe}$ ratio of Population III supernovae with the $v$-process reproduces the corresponding range observed in EMP stars. On the other hand, the $\mathrm{Sc} / \mathrm{Fe}$ and $\mathrm{Co} / \mathrm{Fe}$ ratios of Population III supernovae and hypernova are smaller than the observed ratios even if the $v$-process is taken into account.

\section{References}

[1] R. Cayrel et al., Astron. Astrophys. 416 (2004) 405.

[2] K. Nomoto, N. Tominaga, H. Umeda, C. Kobayashi, \& K. Maeda, Nucl. Phys. A 777 (2006) 424.

[3] C. Kobayashi, H. Umeda, K. Nomoto, N. Tominaga, \& T. Ohkubo, Astrophys. J. 653 (2006) 1145.

[4] N. Tominaga, H. Umeda, \& K. Nomoto, Astrophys. J. 660 (2007) 516.

[5] S.E. Woosley, D.H. Hartmann, R.D. Hoffman, \& W.C. Haxton, Astrophys. J. 356272.

[6] A. Heger, E. Kolbe, W.C. Haxton, K. Langanke, G. Martínez-Pinedo, \& S.E. Woosley, Phys. Lett. B 606 (2005) 258.

[7] T. Yoshida, T. Kajino, D.H. Hartmann, Phys. Rev. Lett. 94 (2005) 231101.

[8] T. Yoshida, T. Kajino, H. Yokomakura, K. Kimura, A. Takamura, \& D.H. Hartmann, Phys. Rev. Lett. 96 (2006) 091101.

[9] T. Yoshida, T. Kajino, H. Yokomakura, K. Kimura, A. Takamura, \& D.H. Hartmann, Astrophys. J. 649 (2006) 319.

[10] T. Yoshida, H. Umeda, \& K. Nomoto, Astrophys. J. 672 (2008) 1043.

[11] H. Umeda \& K. Nomoto, Astrophys. J. 565 (2002) 385.

[12] H. Umeda \& K. Nomoto, Astrophys. J. 619 (2005) 427.

[13] T. Yoshida, H. Umeda, \& K. Nomoto, Astrophys. J. 631 (2005) 1039.

[14] C. Fröhlich, et al., Astrophys. J. 637 (2006) 415.

[15] K. Maeda \& K. Nomoto, Astrophys. J. 598 (2003) 1163. 